\title{
A Wavelet Approach for Identification of Linear Time Invariant System
}

\author{
Ramesh Kumar \\ Phd,Associate Professor \\ NIT Patna
}

\author{
Chitranjan Kumar \\ Assistant Professor \\ PSCET, Vaishali
}

\author{
Kumari Arti \\ M.Tech pursuing \\ NIT Patna
}

\begin{abstract}
Wavelet transformation has been applied to various problems of system identification. In this paper, a wavelet based approach for the identification of time-invariant system is proposed. In this approach, mother wavelet is used for excitation to find the impulse response, which can be estimated at half the available number of points of the sampled output sequence. This method has been compared with some other standard techniques such as frequency chirp, coherence function and inverse filtering. In chirp method, wideband excitation such as frequency chirp is used. Frequency response is obtained as the DFT of the output of the system for time-domain input. Inverse method uses SVD function to find pseudoinverse. Coherence function has been used to identify the system using MATLAB function tfestimate. The performances of the methods are demonstrated by means of experimental investigation.
\end{abstract}

\section{Keywords: WAVELET, SUT, SYSTEM IDENTIFICATION}

\section{INTRODUCTION}

Many engineering applications need a compact and accurate description of system's behavior. Various methods for the system identification has been developed in last recent years. Some of the techniques are given in[1],[2]. Wavelets have been applied for non-linear[3-9]/parametric as well as timevarying system[9-13]. Wavelet transformation provides timescale information of a signal. It has applications like signal detection, adaptive filtering, image processing, denoising, compression[14-18]. In case of time-invariant, it is very difficult to find relation between Discrete Wavelet Transform (DWT) and time-invariant signals, because DWTs are not time-invariant. Here, mother wavelet has been used to excite the SUT. DWT of output of SUT will give the impulse response of that system. Wavelet method has been compared with basic methods. This paper is focused on a wavelet based approach for the identification of time-invariant systems.

\section{WAVELET METHOD}

A signal is represented by following equation

$$
f(t)=\sum_{k, m} a_{k, m} \psi_{k, m}(t), \quad k, m \in \mathbf{Z}
$$

Above eqn is called wavelet in which $\alpha_{k, m}$ is called Discrete Parameter Wavelet Transform (DPWT). DPWT is computed by the following equation:

$$
a_{k, m}=\int f(t) \Psi_{k, m}(t) d t=f(t), \Psi_{k, m}(t) \text {. }
$$

According to above equation, DPWT is an inner product of the signal and the corresponding wavelet. If the signal $f(t)$ and analyzing wavelet have same wavelet, i.e. $f(t)=\Psi_{\alpha, \beta}(t)$ then coefficients of the DPWT of $f(t)$ are as

$a_{k, m}=\Psi_{\alpha, \beta}(t), \Psi_{k, m}(t)$.
For orthogonal wavelets, alpha should be equal to $\mathrm{k}$ so that coefficients do not vanish.

Analysis of the signal corresponds to a multiresolution decomposition of a particular form that allows perfect reconstruction of the signal under certain conditions. For perfect reconstruction, the synthesis filters $g(n)$ and $h(n)$ corresponding to the decomposition filters $h(n)$ and $g(n)$ must satisfy QMFs conditions. These conditions yield two equations:

$$
\begin{aligned}
& \phi(t)=2 \sum_{n} g(n) \phi(2 t-n), \\
& \Psi(t)=2 \sum_{n} h(n) \phi(2 t-n),
\end{aligned}
$$

which are the scaling and wavelet equations, respectively. Here $h(n)$ is not the impulse response of the SUT. The factor of 2 is a scaling or normalizing term.

For time invariant source filter model, the output signal is the convolution of the source signal $x(t)$ and the filter impulse response $h(t)$ :

$y(t)=\int x(\tau) h(t-\tau) d \tau$

The DPWT of $y(t)$ is then

$a_{k, m}=\iint \Psi_{k, m}(t) x(\tau) h(t-\tau) d \tau d t$.

It can be calculated as

$$
a_{k, m}=A h\left(\frac{m-\beta}{2^{k}}\right) \text {. }
$$

Where $A=B C . B$ is constant and $C$ is constant of integration. The impulse response can be expressed in terms of wavelet coefficients as

$h\left(\frac{m}{2^{k}}\right)=\frac{a_{k, m}+\beta}{A}$

Impulse responses can be computed for different values of $k$, such as $k<0, k>0, k=0$. If $k<0$, then the impulse response is decimated by $2^{|k|}$. For example, if $k=-1$ and $S$ coefficients are computed, the impulse response values are (setting $\beta=0$ for simplicity):

$$
\left\{h(0)=\frac{a_{-1,0}}{A}, h(2)=\frac{a_{-1,1}}{A}, h(4)=\frac{a_{-1,2}}{A}, h(6)=\frac{a_{-1,3}}{A}, \ldots, h(2 S)=\frac{a_{-1, S}}{A}\right\},
$$

Thus, aliasing can occur if $k$ is not well chosen. Alternatively, if $k>0$, then it is the sequence of wavelet coefficient values (rather than the impulse response) that is decimated. For example, if $k=1$, the impulse response values are(again with $\beta=0)$ :

$$
\left\{h(0)=\frac{a_{1,0}}{A}, h(1)=\frac{a_{1,2}}{A}, h(2)=\frac{a_{1,4}}{A}, h(3)=\frac{a_{1,6}}{A}, \ldots, h\left(\frac{\zeta}{2}\right)=\frac{a_{1, \zeta}}{A}\right\},
$$

Where $\zeta=2[S / 2]$. A minor concern here is that many wavelet coefficients are not used to determine the impulse response, 
wasting computation time and memory somewhat. In addition, since only the finite number of $S$ wavelet coefficients were obtained in practice, to achieve a good estimate of the impulse response, the later should decay to

zero over $2^{-k} S$ samples. This sequence is much shorter than the number of available samples $S$, again wasting resources. It seems that the best choice of $k=\alpha$ is zero. In some sense, this is intuitively evident, since it corresponds to choice of the mother wavelet as the source signal.

$K=0$ is the best choice to estimate impulse response as

$h(m)=\frac{a_{0, m+\beta}}{A}$.

Then, the frequency characteristic of the SUT is simply the Discrete Fourier Transform (DFT) of the DPWT coefficients for $k=0$ :

$H(n)=\frac{1}{A} \sum_{m=0}^{S-1} a_{0, m+\beta} e^{-2 \pi j m n / S}$.

In summary, an attractive approach to system identification is to excite the SUT with some time-compact function satisfying the orthogonality condition (5), whereupon the system impulse (or frequency) response should be recoverable from the coefficients of the wavelet transform of the output, using the form of the input excitation as the wavelet transform analyzing function.

The continuous analysis above carries over to the discrete case as follows:

DWT synthesis:

$y(n)=\sum_{k, m} a_{k, m} \Psi_{k, m}(n)$,

DWT analysis:

$a_{k, m}=\sum_{n} y(n) \Psi_{k, m}(n)$,

Scaling (dyadic):

$\Psi_{k, m}(n)=B \Psi\left(2^{k} n+m\right)$.

The coefficients of the DWT are given by the discrete wavelet version of Eq. (7), which has precisely the same form as before:

$a_{k, m}=A h\left(\frac{m-\beta}{2^{k}}\right)$

and by the previous argument, $k=0$ is the best choice to prevent aliasing without wasting resources.

\subsection{System Identification Using Discrete Wavelet Transform}

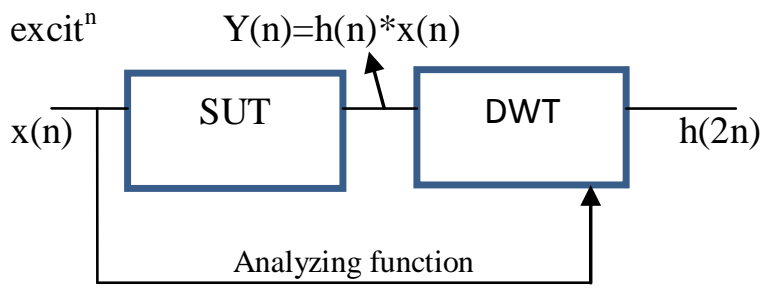

Fig 1.system identification with discrete wavelet transform

SUT is excited by some input to produce output sequence $y(n)$. we then take the DWT of the output signal using the input itself as the analyzing function. Then according to the discrete-time version of Eq. (7), the system impulse response (and hence the frequency response) can be estimated directly from the DWT coefficients, $a_{0, m}$.

\section{RESULT AND DISCUSSION}

\section{Wavelet method}

Two excitations have been taken to verify the utility of the method and chebyshev,iir, 10th-order high pass filter with 20 $d b$ ripple has been taken as system under test (SUT). Excitations are :

Haar $h_{0,0}(n)= \begin{cases}\frac{1}{\sqrt{2}}, & n=0 \\ -\frac{1}{\sqrt{2}}, & n=1 \\ 0, & \text { otherwise, }\end{cases}$
Daubechies $D 4=\left\{\begin{array}{ll}\frac{1-\sqrt{3}}{4 \sqrt{2}},-\frac{3-\sqrt{3}}{4 \sqrt{2}}, \frac{3-\sqrt{3}}{4 \sqrt{2}},-\frac{1-\sqrt{3}}{4 \sqrt{3}}\end{array}\right\}$

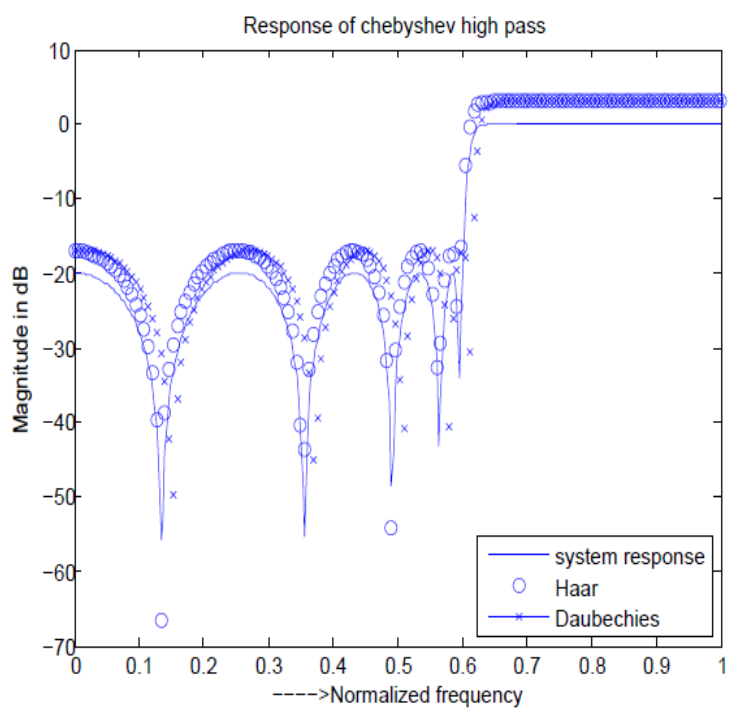

Fig 2. Frequency Response of chebyshev high pass filter using wavelet (Haar and Daubechies) method

Frequency response of chebyshev high pass filter is shown in above figure using a 256-point Fast Fourier Transform (FFT),so that the system response is identified at 128 points. From fig. it can be seen that the Haar and Daubechies excitations give very good identification

\section{COMPARISON WITH CONVENTIONAL SYSTEM IDENTIFICATION METHODS}

\subsection{Chirp method}

Identification can be achieved using a wideband excitation such as frequeney chirp (swept sinuoid). Frequency response is obtained as the Discrete Fourier Transform of the output of the system for such a time-domain input. For low pass filter responses, this method works well but a chirp signal has poor high frequency content and this affects the identification. Below fig. shows the identification of the chebyshev high pass filter response by wavelet based method and chirp method using a 256-point FFT and the DFT. The superiority of the wavelet identification is easily apparent. 


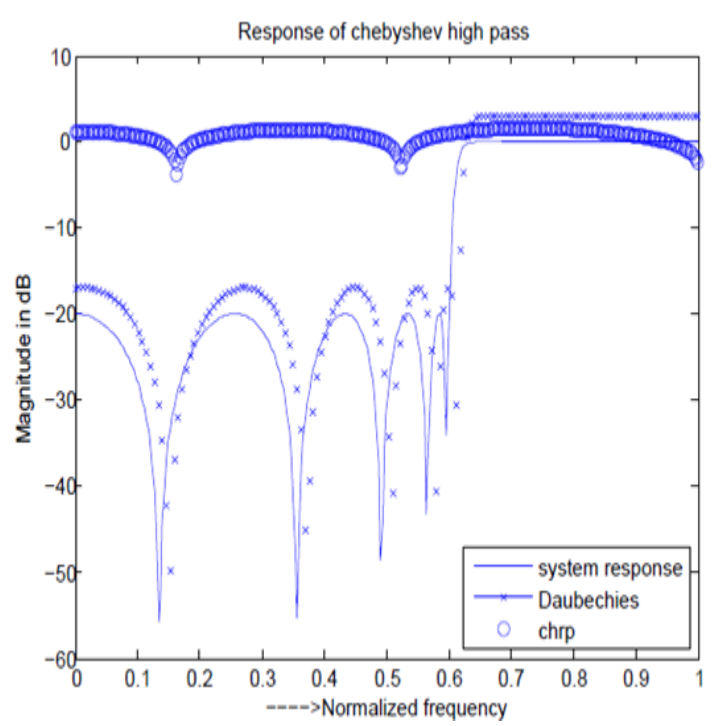

Fig 3.Response of chebyshev high pass filter using chirp and daubechies (wavelet) methods.

\subsection{Inverse filtering method}

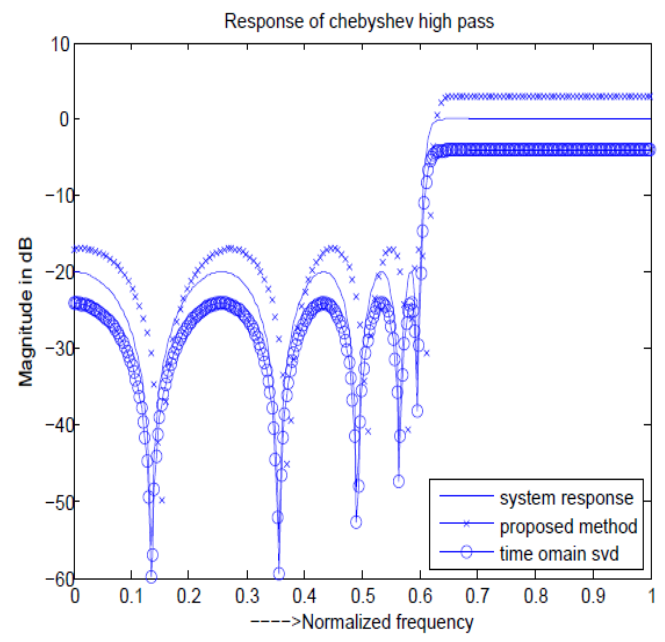

Fig 4. Response of chebyshev high pass filter using inverse filtering and daubechies (proposed) methods.

The pseudoinverse is found by singular value decomposition using MATLAB function svd. The input excitation for the SVD identification was a pseudorandom sequence of length 1000 samples (truncated to 512 points for subsequent FFT processing to find the system function). SVD minimizes the squared error between the output sequence and the convolution of the input with the identified system response. Figure shows the comparison of the wavelet based method with time-domain SVD for our example Chebyshev high-pass filter. Here, the wavelet-based method outperforms SVD for identification in the pass band and remains reasonable provided we do not extend too far into the stop band. SVD identification could be made as good as the wavelet-based method by using the full rank of the matrices. However, this takes quite a long time to perform (several minutes with a Sun Ultrasparc as opposed to less than a second for the proposed method).

\subsection{Coherence method}

Coherence equation has been used to identify the Chebyshev high-pass filter using MATLAB function tfe (transfer function estimate) from the signal processing toolbox. The input excitation for the coherence identification was a pseudorandom sequence of 10,000 samples (truncated to 512 points for subsequent FFT processing to find the system function). Figure shows the comparative results, with the wavelet-based method gain proving superior.

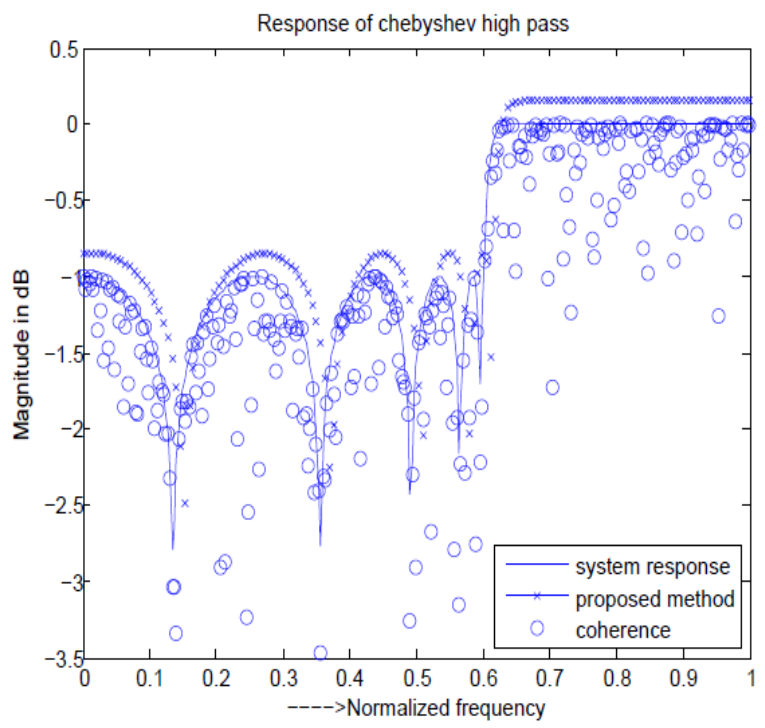

Fig 5. Response of chebyshev high pass filter using coherence method and daubechies (proposed) methods.

\section{CONCLUSION}

Comparing chirp method, inverse filtering and coherence method and taking frequency response of system as reference, inverse filtering is found to be more suitable for system identification. However, it takes a long time to perform.

For wavelet method, Mother Wavelet is used for excitation for SUT. Mother wavelet also acts as analyzing function for Discrete Wavelet Transform (DWT). Impulse response is achieved by converting the output in wavelet transform at half the available number of points of sampled output sequence.

Wavelet method is compared with basic methods named as chirp method, inverse filtering and coherence method. Identification has been carried out for infinite impulse response system and identified that wavelet based method is better than other methods.

\section{REFERENCES}

[1] D. J. Ewins. Modal: Theory, Practice and applications. Engineering Dynamic Series. Research Studies press Ltd., Baldock, Hertfordshire, England, second edition, 2000 .

[2] D. J. Ewins and D. J. Inmam, editors. Structural Dynamics 2000: current direction and future detection. Engineering Dynamic Series. Research Studies press Ltd., Baldock, Hertfordshire, England,2001 
[3] A. Juditsky, Q. Zhang, B. Delyon, P.-Y.Glorennec, A. Benveniste, Wavelets in identification, IRISA Publication No. 849, September 1994.

[4] M. Pawlak, Z. Hasiewicz, Nonlinear system identification by the Haar multiresolution analysis, IEEE Trans. Circuits Systems I-Fundam. Theory Appl. 45 (9) (1998) 945-961.

[5] W.J. Staszewski, Identification of non-linear systems using multi-scale ridges and skeletons of the wavelet transform, J. Sound Vibrat. 214 (4) (1998) 639-658.

[6] W.J. Staszewski, Analysis of non-linear systems using wavelets, Proc. Inst. Mech. Eng. C 214 (11) (2000) $1339-1353$.

[7] D. Coca, S.A. Billings, Non-linear system identification using wavelet multiresolution models, Int. J. Control 74 (18) (2001) 1718-1736.

[8] R. Ghanem, F. Romeo, A wavelet-based approach for model and parameter identification of non-linear systems, Int. J. Nonlinear Mech. 36 (5) (2001) 835-859.

[9] M. Pawlak, Z. Hasiewicz, Nonlinear system identification by the Haar multiresolution analysis, IEEE Trans. Circuits Systems I-Fundam. Theory Appl. 45 (9) (1998) 945-961.

[10] A.N. Robertson, K.C. Park, K.F. Alvin, Extraction of impulse response data via wavelet transform for structural system identification, J. Vibrat. Acoust. Trans. ASME-120 (1) (1998) 252-260.

[11] M.K. Tsatanis, G.B. Giannakis, Time-varying system identification and model validation using wavelets, IEEE Trans. Signal Process. 41 (12) (1993) 3512-3523.

[12] M.I. Doroslova cki, H. Fan, L. Yao,Wavelet-based identification of linear discrete-time systems: Robustness issues, Automatica 34 (12) (1998) 1637-1640.

[13] Y. Zheng, Z. Lin, D.B.H. Tay, Time-varying parametric system multiresolution identification by wavelets, Int. J. Syst. Sci. 32 (6) (2001)

[14] H. Guo, C.S. Burrus, Wavelet and image compression using the Burrows Wheeler transform and the wavelet transform, in: Proceedings of the IEEE International Conference on Image Processing, ICIP-97, vol. I, Santa Barbara, CA, October 1997, pp. 65-68.
[15] D.L. Donoho, De-noising by soft-thresholding, IEEE Trans. Inform. Theory 41 (3) (1995) 613-627.

[16] S. Del Marco, J.Weiss, Improved transient signal detection using a wave packet-based detector with an extended translation-invariant wavelet transform, IEEE Trans. Signal Process. 45 (4) (1997) 841-850.

[17] N. Erdol, F. Basbug,Wavelet transform based adaptive filtering, in: J. Vandewalle, R. Boite, M. Moone, A. Oosterlinck (Eds.), Signal Processing VI: Theories and Applications, Elsevier, Amsterdam, 1992, pp. 1117 1120.

[18] S. Hosur, A.H. Tewik, Wavelet transform domain LMS algorithm, in: Proceedings of the IEEE International Conference on Acoustics, Speech and Signal Processing, vol. 3, Minneapolis, MN, April 1993, pp. 508-510.775793.

[19] C.S. Burrus, R.A. Gopinath, H. Guo, Introduction to Wavelets and Wavelet Transforms: A Primer, Prentice Hall, Upper Saddle River, NJ, 1997.

\section{AUTHORS PROFILE}

Dr. Ramesh Kumar received the B.Sc (Engg.), Electrical, at B.C.E/NIT Patna,theM.Sc degree in Electrical Engg\&Ph.D from Patna University,patna. $\mathrm{He}$ is now Associate Professor in Department of Electrical Engg.at NIT Patna.

Chitranjan Kumar obtained Bachelor of Engineering (B.E) degree in Electronics and Telecommunication engineering from the D.K.T.E.S, Ichalkaranji, Shivaji University, Kolhapur, and M.Tech in Control System, Department of Electrical Engineering at NIT Patna.

Kumara Arti received B.Tech degree in Electronics and Communication Engineering from Rajsthan Technical University, Jaipur and pursuing M.Tech from NIT Patna in Control System. She has two years of teaching experience. 